\title{
DFT Study on Structural and Mechanical Properties of Single-walled Carbon and Boron Nitride Nanotubes Functionalized with Carbenes
}

\author{
I.K. Petrushenko* \\ Physical and Technical Institute, Irkutsk National Research Technical University, 83, Lermontov st., 664074 \\ Irkutsk, Russia
}

(Received 04 May 2016; published online 03 October 2016)

\begin{abstract}
This paper presents quantum chemistry study on structural and mechanical properties of a series of single-walled carbon nanotubes (SWCNTs) and boron nitride nanotubes (BNNTs) functionalized with carbenes. At the PBE/SVP level, the obtained data on pristine nanotubes are in good accordance with the results of previous experimental and theoretical studies. The calculations show that carbenes functionalization, in general, distorts both SWNCTs and BNNTs frameworks, but there exists the difference between 'axial' and 'circumferential' functionalization. It turns out that in both cases elastic properties diminish with increasing concentration of adsorbents, however, the functionalized SWCNTs and BNNTs remain strong enough to be suitable for reinforcement of composites.
\end{abstract}

Keywords: DFT, PBE, Single-walled carbon nanotube, Boron nitride nanotube, Young's modulus.

DOI: 10.21272/jnep.8(3).03031

PACS numbers: 31.15.E -, 61.48. - c, 62.20.de, $71.15 . \mathrm{Mb}$

\section{INTRODUCTION}

Since the Iijima's discovery [1], single-walled carbon nanotubes (SWCNTs) have become a focus of theoretical and experimental studies owing to their perfect structures and unique properties as well as potential applications. SWCNTs demonstrate outstanding mechanical, physical, chemical, thermal, electronic, and optical properties, which predetermine their great influence on nanotechnologies [2-9]. It was found that their Young's modulus is $\sim 1 \mathrm{TPa}$ [6]. However, there exist some deviations of Young's modulus values both experimentally obtained and theoretically predicted. They are caused by many reasons, such as various computational techniques used, different methods of determination of nanotubes (NTs) walls thickness and so on. Summarized data on the mechanical properties of SWCNTs can be found elsewhere [10]. At the same time, the usage of pristine SWCNTs does not allow one to incorporate them effectively in composite materials for the purposes of strengthening. It is very important to bind strongly the host matrix and reinforcement materials as well as prevent agglomeration of SWCNTs. To overcome this problem, researchers use various functionalization techniques to bind functional groups on the walls of NTs and promote their further derivatization.

Boron nitride nanotubes (BNNTs) were theoretically predicted in 1994, and it is not until 1995 that scientists succeeded in obtaining them [11]. BNNTs exhibit excellent mechanical properties and high thermal conductivity as structural analogues of SWCNTs. They also possess very high Young's modulus of $\sim 0.7$ $1.0 \mathrm{TPa}$ [12]. Therefore, BNNTs can be considered as promising novel materials for nanoelectronics. At the same time, their electronic properties are significantly differ from those of SWCNTs. Unlike the latter, BNNTs are always semiconductors with a wide band gap, which depends slightly on their diameters and chirali- ties [11]. However, their tendency to aggregation and insolubility effects slow down the potential application of BNNTs in micro- and nanoelectromechanical systems. As in the case of SWCNTs, the usage of pristine BNNTs is rather limited and it is worth studying mechanical properties of functionalized NTs.

Carbenes $\left(\mathrm{CR}_{2}, \mathrm{R}=\mathrm{H}, \mathrm{F}, \mathrm{Cl}, \mathrm{CH}_{3}, \mathrm{CN}\right.$, and $\left.\mathrm{NO}_{2}\right)$ are divalent carbon species. Recently, the addition of carbenes on fullerenes and NTs has been thoroughly studied. Most of the works reported the $[2+1]$ cycloaddition of dichlorocarbene $\left(\mathrm{CCl}_{2}\right)$ on sidewalls of both SWCNTs $[13,14]$ and BNNTs $[15,16]$, whereas a few papers investigated carbenes addition on fullerenes [17] and graphene [18]. Thus, Luksirikul et al., using the high reactivity of carbenes, obtained functionalized SWCNTs with different substituents; the authors pointed out that they enhance dispersibility of such SWCNTs [19]. From this point of view, we can assume the $\mathrm{CH}_{2}$ functionalization of SWCNTs and BNNTs, owing to its simplicity, as a good starting point for a novel nanotube/polymer composite design. Therefore, a study of the structural and mechanical properties of SWCNT and BNNT models functionalized by $\mathrm{CH}_{2}$ was undertaken.

In fact, owing to limitations of the current experimental methods, there is a lack of fundamental data on the elastic modulus of various types of functionalized SWCNTs. At the same time, the number of papers on mechanical properties of BNNTs is much more modest. In light of our continuing interest on studying mechanical and structural properties of SWCNTs [2, 3, 20, 21], we perform a comparative DFT study on CH2 functionalization of finite-size SWCNTs and BNNTs, focusing on equilibrium structures of the $\mathrm{CH} 2$-attached NTs and the effect of the degree of functionalization on the elastic properties of such SWCNTs and BNNTs.

\section{COMPUTATIONAL METHODS}

\footnotetext{
* igor.petrushenko@istu.edu
} 
The structural models $\mathrm{C}_{60} \mathrm{H}_{20}$ were used as SWCNTs; the models $\mathrm{B}_{30} \mathrm{~N}_{30} \mathrm{H}_{20}$ were utilized to represent BNNTs. The ends of all NTs were saturated by hydrogen atoms to avoid the dangling. For all calculations we used Orca 3.0.3 program package [22]. DFT method with the PBE functional was employed $[23,24]$. The PBE has been demonstrated to be a reliable and commonly used functional for study of different nanostructures [25-27]. To calculate equilibrium geometries of studied structures we used Ahlirchs' SVP (split-valence polarization) basis set [28]. In SVP the inner shell atomic orbitals are described by a single basis function, two basis functions are provided for each valence shell atomic orbital, augmented by a set of polarization functions.

In order to calculate Young's modulus of every model we used the following formula:

$$
Y=\sigma \varepsilon=2 \times(\Delta E / S) \times(L /(\Delta L) 2)
$$

where $\sigma$ is the stress, $\varepsilon$ is the strain, $E$ is the increment of the total energy, $S$ is the area upon which stretching force acts, $L$ is the length of a tube and $\Delta L$ is the elongation upon stretching. The $S$ area can be calculated by the formula: $S=2 \pi a r$, where $r$ is the radius of the NT, $a=3.4 \AA$, which is equal to the graphite interlayer distance. In the case of BNNTs, we also selected $a=3.4 \AA$ following the results of Hod [29].

Binding energy $\left(E_{b}\right)$ of a carbene $\left(\mathrm{CH}_{2}\right)$ molecule with the NT (SWCNT or BNNT) was defined as follows:

$$
E_{b}=\left[E\left(n \mathrm{CH}_{2} / \mathrm{NT}\right)-E(\mathrm{NT})-n E\left(\mathrm{CH}_{2}\right)\right] / n,
$$

where $E\left(\mathrm{CCl}_{2} / \mathrm{NT}\right)$ is the total energy of carbene adsorbed on a NT surface; $E(\mathrm{NT})$ and $E\left(\mathrm{H}_{2}\right)$ are the total energies of a pristine NT and an isolated carbene molecule; $n$ stands for the number of attached $\mathrm{CH}_{2}$.

It is worth saying that most studies on NTs should imply more complicated techniques than just cluster calculations, for instance, electronic properties calculations. However, to obtain the elastic moduli researchers often use simpler models that produce almost the same results as in the case of periodic calculations and huge cluster structures. Such has indeed proved to be the case, as we shall see in the following sections. Therefore, in this work, we employ cluster models of NTs.

\section{RESULTS AND DISCUSSIONS}

\subsection{Structural Properties of $\mathrm{CH}_{2}$-attached SWCNTs}

Firstly, we considered two possible positions for the attachment of an individual carbene molecule on the sidewall of SWCNTs. According to the previous investigations [2, 3, 30, 31], one can select two different types of C-C bonds in SWCNTs where the attachment can occurs. The first of them is labelled as 'axial' (b1), the second is 'circumferential' (b2) (Fig. 1).

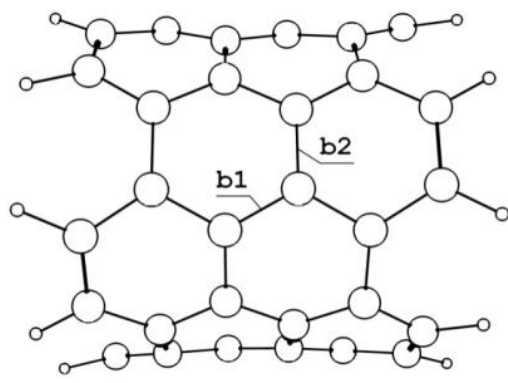

Fig. 1 - Optimized structure of SWCNT. C and H atoms are represented by big and small balls, respectively. Two C-C bonds on SWCNT are labelled as 'axial' (b1) and 'circumferential' (b2)

Actually, the angle between b1 and the axes of NTs is actually $\sim 30^{\circ}$. We use such notations to highlight the fundamental difference of these bonds. Secondly, we studied the most stable configuration of the $\mathrm{CH}_{2}$ decorated NTs for these two attachment sites. The bond lengths and binding energies of functionalized NTs with various coverage by $\mathrm{CH}_{2}$ molecules are summarized in Table 1.

Table 1 shows that the b2 bond is favourable for $\mathrm{CH}_{2}$ functionalization, however, we still should note that the difference of $E_{\mathrm{b}}$ for b1 and b2 is rather small. Our results coincide well with the previous results of Bettinger [13]. In this paper, therefore, we will study the b2 cycloaddition only. Despite the fact that the $\mathrm{CH}_{2}$ opens the b2 bond leaving the b1 bond almost intact, the higher local curvature stands for the $\mathrm{b} 2$ bonds are more reactive than $b 1$ ones. The functionalization of SWCNT with higher $\mathrm{CH}_{2}$ coverage was also investigated. We increase the number of $\mathrm{CH} 2$ molecules on the sidewall of the SWCNT from 1 to 5 .

Herein, the density of coverage is defined as the number of bonds that $\mathrm{CH}_{2}$ make with the carbon atoms of the SWCNT vs. the total number of carbon atoms in our model. Therefore, according to the studied models, one $\mathrm{CH}_{2}$ corresponds to the coverage of $5 \%$, five $\mathrm{CH}_{2}$ molecules correspond to the coverage of $25 \%$. Initially, the first studied addend was attached on the 'circumferential' C-C bond as it is energetically favoured. The method of further SWCNTs decoration was as follows: the second $\mathrm{CH}_{2}$ species was placed on the 'circumferential' C-C bond as far as possible from the first addend, which dictate the least interaction between them. The third, fourth, and fifth species were also linked to the 'circumferential' $\mathrm{C}-\mathrm{C}$ bonds in such a way to cover the surface of the SWCNT uniformly. The binding energy for one $\mathrm{CH}_{2}$ is $-4.939 \mathrm{eV}$, the further functionalization gives modest impact in the $E_{b}$ values (Table 1).

In terms of the thermal stability, the more negative values demonstrate the ease of functionalization with carbene. The length of $\mathrm{C}-\mathrm{C}$ bonds underlying carbene is elongated only marginally on going from one to five carbene species (Table 1). 
Table 1 - The bond lengths $\left(d_{c-c}\right)$ and binding energies $\left(E_{\mathrm{b}}\right)$ of functionalized SWCNTs and BNNTs with various coverages by $\mathrm{CH}_{2}$

\begin{tabular}{|c|c|c|c|c|c|c|}
\hline & \multicolumn{3}{|l|}{ SWCNTs } & \multicolumn{3}{|l|}{ BNNTs } \\
\hline Addends & Coverage, $\%$ & $d_{\mathrm{C}-\mathrm{C}, \AA} \AA$ & $E_{b}, \mathrm{eV}$ & Coverage, $\%$ & $d$ B-N,$\AA$ & $E_{b}, \mathrm{eV}$ \\
\hline- & 0 & $1.444^{\mathrm{a}}, 1.434^{\mathrm{b}}$ & - & 0 & $1.458^{\mathrm{a}}, 1.451^{\mathrm{b}}$ & - \\
\hline $\mathrm{CH}_{2}{ }^{\mathrm{c}}$ & 5 & 1.624 & -3.195 & 5 & 1.701 & -3.044 \\
\hline $\mathrm{CH}_{2}{ }^{\mathrm{d}}$ & 5 & 2.207 & -4.939 & 5 & 2.259 & -4.168 \\
\hline $2 \mathrm{CH}_{2}$ & 10 & $2.202 ; 2.202$ & -4.984 & 10 & $2.257 ; 2.251$ & -4.203 \\
\hline $3 \mathrm{CH}_{2}$ & 15 & $\begin{array}{l}2.199 ; \quad 2.220 ; \\
2.219\end{array}$ & -4.904 & 15 & $2.278 ; 2.276 ; 2.249$ & -4.153 \\
\hline $4 \mathrm{CH}_{2}$ & 20 & $\begin{array}{l}2.213 ; 2.213 ; \\
2.231 ; 2.230\end{array}$ & -4.782 & 20 & $\begin{array}{l}2.294 ; 2.293 ; 2.273 ; \\
2.272\end{array}$ & -4.078 \\
\hline $5 \mathrm{CH}_{2}$ & 25 & $\begin{array}{l}2.219 ; 2.218 ; \\
2.217 ; \quad 2.217 ; \\
2.217\end{array}$ & -4.671 & 25 & $\begin{array}{l}2.290 ; 2.289 ; 2.292 ; \\
2.292 ; 2.290\end{array}$ & -4.040 \\
\hline
\end{tabular}

a'axial' C-C bond

b'circumferential'C-C bond

${ }^{\circ} \mathrm{CH}_{2}$ attachementto the 'axial' (b1) bond,

${ }^{\mathrm{d}} \mathrm{CH}_{2}$ attachementto the 'circumferential' (b2) bond.

Analyzing Table 1 data we can see that two $\mathrm{CH}_{2}$ species attached to the SWCNT surface make the energetically favorable $\mathrm{CH}_{2} / \mathrm{SWCNT}$ system with energy of $-4.984 \mathrm{eV}$. In this case, the $\mathrm{C}-\mathrm{C}$ distances are minimum among studied (2.202 ̊). Here, we use term 'C-C distance' rather than ' $\mathrm{C}-\mathrm{C}$ bond' to underline the fact that the bonds are actually broken. The $\mathrm{CH}_{2}$ addition, generally, induces deformations in the perfect framework of pristine SWCNTs. The cross-section of functionalized SWCNTs is no longer circular but rather elliptic (for 1-4 attached species). However, uniform coverage of SWCNTs with five $\mathrm{CH}_{2}$ molecules restores the circular shape of its cross-section, and C-C distances underlying the attached carbenes become almost equal (Table 1).

Also, one cannot say that binding energy sharply decrease with the increase in $\mathrm{CH}_{2}$ coverage. In fact, the curvature of the SWCNT's walls remains the same on the gradual functionalization and the practically equal obtained values of $E_{b}$ demonstrate it clearly (Table 1). This is in contradictory with previously reported results on cycloaddition of various species on graphene sheets [18, 32]. In those works, one can observe the strong dependence of $E_{\mathrm{b}}$ regarding the position of functionalization.

It should be noted, even at the highest studied degree of functionalization of $25 \%$, the binding energy value is still large, which suggests a good possibility of further functionalization.

\subsection{Structural Properties of CH2-attached BNNTs}

In this section, by analogy with the aforementioned discussion, we considered two possible positions for the attachment of an individual carbene molecule on the sidewall of BNNTs. They can be considered as rolled-up sheets of hexagonal boron nitride $(\mathrm{BN})$. In terms of structures, a single layer of $\mathrm{BN}$ is very similar to a graphene sheet having a hexagonal skeleton where each pair of bonded carbon atoms is replaced by a boron-nitride pair. Nevertheless, due to the electronegativity differences be- tween the atoms of boron and nitrogen the $\pi$-electrons tend to localize around the nitrogen atomic centers. Despite the fundamental difference between C-C and B-N bonds, we still observe the same behaviour on $\mathrm{CH}_{2}$ attachment: i) favorable 'circumferential' addition and ii) two $\mathrm{CH}_{2}$ species linked to the SWCNT surface make the energetically favorable $\mathrm{CH}_{2} / \mathrm{BNNT}$ system with energy of $-4.203 \mathrm{eV}$ (Table 1). The method of BNNTs decoration as well as the definition of $\mathrm{CH}_{2}$ coverage were the same as in the case of SWCNTs. The length of B-N bonds underlying carbene is also elongated only marginally on going from one to five carbene species. In general, the functionalization of SWCNTs by carbenes is more energetically favorable than that of BNNTs. The latter is also characterized by the large values of binding energy, which suggests a good possibility of further functionalization even in the case of $25 \%$ coverage.

To sum up, functionalization of both SWCNTs and BNNTs is exothermic. All studied reactions are spontaneous, although the binding energies on 'circumferential' and 'axial' functionalization are different.

\subsection{Mechanical Properties of Functionalized SWCNTs and BNNTs}

Before we start studying the Young's moduli of the SWCNTs and BNNTs, we would like to present our results for pristine $(5,5)$ SWCNTs. This allows one to compare the results of this paper with previous calculations and to assess the reliability of obtained data. The calculated Young's modulus for the SWCNT (1.02 TPa) corresponds well with the experimental findings including atomic force microscopy $(1.002,1.2$, and $0.8 \pm 0.41 \mathrm{TPa})$ [10,33], transmission electron microscopy (0.9-1.7 and 0.4-4.15 TPa)[10], and micro-Raman spectroscopy studies (2.8-3.6 TPa) [34]. Additionally, we observe the excellent agreement with the previously calculated Young's modulus for the SWCNT. The results of Harik (1.01 TPa) [35], Odegard et al. (0.95-1.1 TPa) [36], Zhang et al. (1.08 TPa) [37], and Milowska and Majewski (1.02 TPa) [38] are almost the same as in this paper. To further validate obtained results, we compare our calculations on BNNTs with recent literature. The calculated Young's modulus for the BNNT $(0.78 \mathrm{TPa})$ is in very good accordance with previous results 0.87-1.11 [27], 0.837-0.912 [13]. Thus, based on the present brief review, our choice of models and methods seems to be justified. 
Table 2 - Young's modulus of functionalized SWCNTs and BNNTs

\begin{tabular}{|c|c|c|c|c|}
\hline \multirow{2}{*}{ Type of NT } & \multicolumn{2}{|c|}{ SWCNT } & \multicolumn{2}{c|}{ BNNT } \\
\hline & $\begin{array}{c}\text { Young's modulus, } \\
\text { TPa }\end{array}$ & Change, $\%$ & Young's modulus, \\
& 1.02 & - & 0.78 & - \\
\hline Pristine NT & 0.82 & 19 & 0.76 & 2 \\
\hline $1 \mathrm{CH}_{2} / \mathrm{NT}$ & 0.66 & 35 & 0.73 & 6 \\
\hline $2 \mathrm{CH}_{2} / \mathrm{NT}$ & 0.57 & 44 & 0.70 & 10 \\
\hline $3 \mathrm{CH}_{2} / \mathrm{NT}$ & 0.56 & 44 & 0.64 & 19 \\
\hline $4 \mathrm{CH}_{2} / \mathrm{NT}$ & 0.72 & 29 & 0.58 & 25 \\
\hline $5 \mathrm{CH}_{2} / \mathrm{NT}$ & & & & \\
\hline
\end{tabular}

Now we present theoretical predictions for elastic characteristics of the SWCNTs and BNNTs (Table 2). As is already mentioned, pristine SWCNTs have larger Young's modulus (1.02 TPa) than their inorganic counterparts $(0.78 \mathrm{TPa})$. NTs decoration with various species leads to the large distortions of their frameworks. It is natural that such a functionalization also causes the Young's modulus decrease (Table 2). In general, the decrease is larger in the case of SWCNTs. For 4 attached carbenes, we may note the maximum drop in SWCNTs Young's modulus of $44 \%$. However, in the case of 5 attached species, we surprisingly observed certain increase in Young's modulus values. We can ascribe this feature to the changes of the cross-section of functionalized SWCNTs. As it was stated above, the functionalization distorts the perfect framework of NTs, and the cross-section adopts elliptic shape (for 1-4 attached species). On the other side, our models provide uniform circumferential coverage of SWCNTs with five attached $\mathrm{CH}_{2}$ molecules. It restores the circular shape of the cross-section, and Young's modulus becomes higher (Table 2). On studying BNNTs, one cannot observe the same feature. The functionalization leads to the gradual, although very moderate, decrease in Young's modulus. The maximum drop of BNNTs elastic modulus is only $25 \%$. The difference of behavior of BNNTs and SWCNTs with 5 attached species

Therefore, one may see that both SWCNTs and BNNTs are promising candidates for the composites reinforcement, although the original characteristics of the latter are rather modest in comparison with SWCNTs. However, the Young's modulus drop upon functionalization is generally smaller for BNNTs.

Thus, the present calculations provide theoretical predictions for the elastic moduli of the functionalized SWCNTs and BNNTs. In the situation of the lack of experimental studies on the BNNTs, both pristine and functionalized, the obtained values should facilitate the understanding and the design of the novel composite materials. Besides this, the data are particularly important for applications which rely on the mechanical properties of individual NTs, such as resonators and sensors [39]. Finally, we suppose that both types of studied functionalized NTs are a good solution to reinforce composite materials.

In summary, the functionalization diminishes the Young's modulus of both SWCNTs and BNNTs. This effect is found to be non-negligible. However, the elastic modulus remains high enough to guarantee successful usage of such NTs for the reinforcement of composites. It is also worth mentioning that Chen et al. [40] have recently designed atomically precise graphene nanoribbons, which gives us firm believe that scientists will soon create other carbon and boron nitride allotropes with atomic accuracy. This is sure helps us to expand our theoretical findings to a practical side.

\section{CONCLUSION}

All in all, we have performed DFT calculations on the addition of $\mathrm{CH}_{2}$ to SWCNTs and BNNTs as well as discussed the structural and elastic properties of $\mathrm{CH}_{2}$ attached NTs. Special attention has been paid to the Young's modulus values of both studied types of NTs. For the studied models, the 'circumferential' C-C bond is preferable for the functionalization. $\mathrm{CH}_{2}$ addition to the 'circumferential' C-C bond leads to its breaking and the formation of open structures on the sidewalls of SWCNTs. It was found that the $E_{b}$ values are very moderately dependent on the number of attached species. Although pristine SWCNTs have larger elastic modulus than BNNTs, the Young's modulus of functionalized SWCNTs decreases notably in comparison with their inorganic counterparts. In the cases of 1-4 attached species, the frameworks of both BNNTs and SWCNTs become strongly distorted. The uniform functionalization with 5 attached species, in turn, makes the shape of studied NTs cross-sections circular. Finally, we propose that BNNTs and SWCNTs both pristine and functionalized are promising candidates for composites reinforcement.

\section{ACKNOWLEDGEMENTS}

IKP thanks Dr. V. Malkin for his continued support and encouragement. IKP thanks SAIA for its support. 


\section{REFERENCES}

1. S. Iijima, Nature 354, 56 (1991).

2. I.K. Petrushenko, N.A. Ivanov, Phys. E 54, 262 (2013).

3. I.K. Petrushenko, N.A. Ivanov, Mod. Phys. Lett. B 27, 1350210 (2013).

4. T. Ando, NPG Asia Mater. 1, 17 (2009).

5. K. Chu, C.-C. Jia, L.-K. Jiang, W.-S. Li, Mater. Design 45, 407 (2013)

6. I. Kumar, S. Rana, J.W. Cho, Chemistry A European Journal 17, 11092 (2011).

7. E.M. Jackson, P.E. Laibinis, W.E. Collins, A. Ueda, C.D. Wingard, B. Penn, Compos. Part B-Engineering 89, 362 (2016).

8. C. Gao, Z. Guo, J.H. Liu, X.J. Huang, Nanoscale 4, 1948 (2012).

9. A. Goldoni, L. Petaccia, S. Lizzit, R. Larciprete, J. PhysCondens. Mat. 22, 013001 (2010).

10. M.M. Shokrieh, R. Rafiee, Mech. Compos. Mater. 46, 155 (2010).

11. A. Rubio, J. Corkill, M. Cohen, Phys. Rev. B 49, 5081 (1994).

12. R. Arenal, M.S. Wang, Z. Xu, A. Loiseau, D. Golberg, Nanotechnology 22, 265704 (2011).

13. H.F. Bettinger, Chem. A Eur. J. 12, 4372 (2006).

14. Y.-Y. Chu, M.-D. Su, Chem. Phys. Lett. 394, 231 (2004).

15. F. Cao, W. Ren, X. Xu, Y.M. Ji, C. Zhao, Phys. Chem. Chem. Phys. 11, 6256 (2009).

16. Y. Li, Z. Zhou, J. Zhao, Nanotechnology 19, 015202 (2008).

17. M. Yamada, T. Akasaka, S. Nagase, Chem. Rev. 113, 7209 (2013).

18. I.K. Petrushenko, Monatsh. Chem. 145, 891 (2014).

19. P. Luksirikul, B. Ballesteros, G. Tobias, M.G. Moloney, M.L.H. Green, J. Mater. Chem. 21, 19080 (2011).

20. I.K. Petrushenko, N.A. Ivanov, Fullerenes, Nanotubes and Carbon Nanostruct. 22, 405 (2014).

21. I.K. Petrushenko, N.A. Ivanov, Fullerenes, Nanotubes and Carbon Nanostruct. 22, 781 (2014).
22. F. Neese, Wires Comput. Mol. Sci. 2, 73 (2012).

23. J.P. Perdew, K. Burke, M. Ernzerhof, Phys. Rev. Lett. 77, 3865 (1996).

24. J.P. Perdew, K. Burke, M. Ernzerhof, Phys. Rev. Lett. 78 , 1396 (1997).

25. I.K. Petrushenko, K.B. Petrushenko, Monatsh. Chem. 146 , 1603 (2015).

26. D.S. Sabirov, A.O. Terentyev, F. Cataldo, Comput. Theor. Chem. 1081, 44 (2016).

27. A.V. Rybalchenko, M.G. Apenova, O.O. Semivrazhskaya, N.M. Belov, V.Y. Markov, S.I. Troyanov, I.N. Ioffe, N.S. Lukonina, L.N. Sidorov, T.V. Magdesieva, A.A. Goryunkov, Electrochim. Acta 191, 980 (2016).

28. A. Schafer, R. Ahlrichs, J. Chem. Phys. 97, 2571 (1992)

29. O. Hod, J. Chem. Theory Comput. 8, 1360 (2012).

30. Z. Zhou, M. Steigerwald, M. Hybertsen, L. Brus, R.A. Friesner, J. Am. Chem. Soc. 126, 3597 (2004).

31. A. Galano, Chem. Phys. 327, 159 (2006).

32. Y. Cao, K.N. Houk, J Mater. Chem. 21, 1503 (2011).

33. J.-P. Salvetat, G. Briggs, J.-M. Bonard, R. Bacsa, A. Kulik, T. Stöckli, N. Burnham, L. Forró, Phys. Rev. Lett. 82, 944 (1999).

34. O. Lourie, D.M. Cox, H.D. Wagner, Phys. Rev. Lett. 81, 1638 (1998).

35. V.M. Harik, Comp. Mater. Sci. 24, 328 (2002)

36. G. Odegard, T.S. Gates, L.M. Nicholson, K.E. Wise, Compos. Sci. Technol. 62, 1869 (2002).

37. H.W. Zhang, J.B. Wang, X. Guo, J Mech. Phys. Solids 53, 1929 (2005).

38. K.Z. Milowska, J.A. Majewski, Phys. Chem. Chem. Phys. 1514303 (2013)

39. Y. Shao, J. Wang, H. Wu, J. Liu, I.A. Aksay, Y. Lin, Electroanal. 22, 1027 (2010).

40. Y.C. Chen, D.G. de Oteyza, Z. Pedramrazi, C. Chen, F.R. Fischer, M.F. Crommie, ACS Nano 7, 6123 (2013). 